\title{
Differential Transition State Stabilization in Enzyme Catalysis: Quantum Chemical Analysis of Interactions in the Chorismate Mutase Reaction and Prediction of the Optimal Catalytic Field
}

\author{
Borys Szefczyk $^{a, *}$, Adrian J. Mulholland ${ }^{b}$, \\ Kara E. Ranaghan ${ }^{b}$ and W. Andrzej Sokalski ${ }^{a, *}$ \\ a Institute of Physical \& Theoretical Chemistry, Wroctaw University of Technology, \\ Wybrzeże Wyspiańskiego 27, 50-370 Wroctaw, Poland \\ ${ }^{b}$ Centre for Computational Chemistry, School of Chemistry, University of Bristol, Bristol \\ BS8 1TS, UK \\ * Authors, to whom correspondence should be addressed; \\ E-mail: borys.szefczyk@pwr.wroc.pl,sokalski@pwr.wroc.pl
}

Supporting Information 
Table SI-1: Decomposition of the reactant and TS interaction energies with active site residues (in kcal/mol). $\Delta E^{(1)}$ is the first-order Heitler-London contribution, $\Delta E_{E L}^{(1)}$ is the first-order electrostatic energy, $\Delta E_{E L \cdot M T P}^{(1)}$ is the electrostatic energy calculated using atomic multipole expansion, $\Delta E_{E L \cdot P E N}^{(1)}$ is the penetration term $\left(\Delta E_{E L}^{(1)}-\Delta E_{E L \cdot M T P}^{(1)}\right), \Delta E_{E X}^{(1)}$ is the firstorder exchange energy, $\Delta E_{D E L}^{(R)}$ is the delocalization term $\left(\Delta E^{S C F}-\Delta E^{(1)}\right), \Delta E^{S C F}$ is the Hartree-Fock energy, $\triangle E_{C O R R}$ is the correction term $\left(\Delta E^{M P 2}-\Delta E^{S C F}\right)$ and $\triangle E^{M P 2}$ is the energy at the MP2 level. Residues are arranged according to the decreasing contribution to the activation barrier lowering at the highest theory (MP2) level. Interaction energies for Glu78 and Cys75 were calculated using the optimal conformation of the Glu78 — chorismate/TS complex.

\begin{tabular}{|c|c|c|c|c|c|c|c|c|c|}
\hline Dimer & $\Delta E^{(1)}$ & $\Delta E_{E L}^{(1)}$ & $\Delta E_{E L \cdot M T P}^{(1)}$ & $\Delta E_{E L \cdot P E N}^{(1)}$ & $\Delta E_{E X}^{(1)}$ & $\Delta E_{D E L}^{(R)}$ & $\Delta E^{S C F}$ & $\Delta E_{\text {CORR }}$ & $\Delta E^{M P 2}$ \\
\hline \multicolumn{10}{|c|}{ Substrate } \\
\hline $\operatorname{Arg} 90$ & -125.04 & -160.40 & -155.09 & -5.31 & 35.36 & -26.41 & -151.45 & -7.92 & -159.37 \\
\hline $\operatorname{Arg} 7$ & -124.62 & -172.04 & -161.41 & -10.63 & 47.42 & -31.12 & -155.74 & -5.37 & -161.11 \\
\hline Glu78 & 88.58 & 78.46 & 81.94 & -3.48 & 10.11 & -7.84 & 80.74 & -0.51 & 80.23 \\
\hline XSOL124 & -1.02 & -17.22 & -12.00 & -5.23 & 16.21 & -5.31 & -6.33 & -2.27 & -8.60 \\
\hline $\operatorname{Arg} 116$ & -95.92 & -97.02 & -96.75 & -0.27 & 1.10 & -4.29 & -100.21 & -1.65 & -101.86 \\
\hline Arg63 & -116.03 & -143.54 & -137.41 & -6.13 & 27.52 & -20.75 & -136.78 & -3.56 & -140.33 \\
\hline Cys75 & 5.04 & -4.22 & -0.41 & -3.81 & 9.26 & -3.72 & 1.32 & -2.28 & -0.96 \\
\hline SOLV7102 & -1.10 & -24.97 & -19.24 & -5.73 & 23.87 & -11.16 & -12.26 & -0.09 & -12.35 \\
\hline XSOL116 & -2.47 & -26.64 & -20.27 & -6.37 & 24.17 & -10.37 & -12.84 & -0.08 & -12.92 \\
\hline Tyr108 & 4.50 & 2.63 & 3.46 & -0.83 & 1.87 & -4.52 & -0.02 & -1.25 & -1.27 \\
\hline Val73 & 9.75 & 4.72 & 6.39 & -1.68 & 5.03 & -2.85 & 6.90 & -3.43 & 3.46 \\
\hline Phe57 & 4.23 & -0.62 & 1.38 & -2.00 & 4.85 & -3.61 & 0.63 & -3.51 & -2.88 \\
\hline Lys60 & -71.56 & -71.56 & -71.56 & 0.00 & 0.00 & -0.69 & -72.25 & 0.22 & -72.03 \\
\hline \multicolumn{10}{|c|}{ Transition State } \\
\hline $\operatorname{Arg} 90$ & -132.22 & -171.40 & -160.32 & -11.08 & 39.18 & -30.90 & -163.12 & -5.31 & -168.43 \\
\hline $\operatorname{Arg} 7$ & -127.43 & -180.39 & -168.16 & -12.23 & 52.96 & -34.97 & -162.40 & -4.60 & -167.01 \\
\hline Glu78 & 77.41 & 77.13 & 77.27 & -0.14 & 0.28 & -2.79 & 74.62 & 2.04 & 76.66 \\
\hline XSOL124 & -1.67 & -23.76 & -17.84 & -5.92 & 22.10 & -8.68 & -10.35 & -0.73 & -11.08 \\
\hline $\operatorname{Arg} 116$ & -98.68 & -99.49 & -99.33 & -0.16 & 0.81 & -5.23 & -103.91 & -0.40 & -104.31 \\
\hline Arg63 & -117.35 & -143.91 & -137.50 & -6.41 & 26.56 & -20.22 & -137.57 & -4.16 & -141.73 \\
\hline Cys75 & 5.61 & -6.23 & -1.44 & -4.79 & 11.84 & -4.23 & 1.38 & -3.15 & -1.77 \\
\hline SOLV7102 & -1.04 & -23.94 & -18.60 & -5.34 & 22.90 & -10.71 & -11.74 & -1.02 & -12.77 \\
\hline XSOL116 & -3.43 & -26.34 & -20.38 & -5.96 & 22.91 & -10.38 & -13.81 & 0.51 & -13.30 \\
\hline Tyr108 & 3.88 & 3.13 & 3.46 & -0.33 & 0.74 & -3.86 & 0.02 & -1.00 & -0.98 \\
\hline Val73 & 10.66 & 5.66 & 7.11 & -1.44 & 5.00 & -2.81 & 7.85 & -3.71 & 4.14 \\
\hline Phe57 & 4.91 & -0.36 & 2.01 & -2.37 & 5.28 & -2.84 & 2.07 & -4.10 & -2.03 \\
\hline Lys60 & -70.26 & -70.26 & -70.26 & 0.00 & 0.00 & -0.67 & -70.93 & 0.29 & -70.64 \\
\hline$\Delta$ & -23.95 & -27.74 & -23.01 & -4.70 & 3.79 & -5.65 & -29.60 & 6.36 & -23.26 \\
\hline
\end{tabular}

